
Edith Catalina

Patino Martinez*

Paula

Almonacid Hurtado***

Armando Lenin

Támara Ayús**:

Universidad de Antioquia, Medellín, Colombia.

Recibido: 21 de abril de 2013

Concepto de evaluación: 12 de julio de 2013

Aprobado: 5 de septiembre de 2013

Artículo de investigación

*Economista de la Universidad de Antioquia. Medellín,

Colombia. Correo electrónico: catalina110@gmail.com

**Economista EAFIT, magíster en Ciencias Económicas de la Universidad de Montreal. Profesor investigador de la Universidad EAFIT, Medellín, Colombia. Correo electrónico: palmona1@eafit.edu.co

*** Economista de la Universidad de Antioquia, especialista en Diseño y Evaluación de Proyectos de la Universidad del Norte, magíster en Finanzas de la Universidad EAFIT. Profesor investigador de la Universidad EAFIT, Medellín, Colombia. Correo electrónico: atamaraa@eafit.edu.co

\section{Política monetaria y precio de activos: un análisis desde la tasa de interés para Colombia de 2003 a 2010}

\section{RESUMEN}

El objetivo principal de este trabajo es analizar la incidencia de la política monetaria colombiana en los precios de los activos de renta fija para el periodo 2003-2010 y comprobar que la vía que esta sigue se asemeja a lo que plantea la teoría tradicional, tomando como referencia la teoría de expectativas puras.

Con el objeto de analizar las relaciones de corto y largo plazo entre los TES y la política monetaria, se desarrolla un modelo econométrico de vector de corrección de errores (VEC) tomando como variable explicada el retorno de bonos con plazo a 6 meses, 1, 5 y 25 años, y como variables explicativas la tasa interbancaria, la inflación y la tasa forward. Los principales resultados obtenidos indican que la política monetaria colombiana incide en los precios de estos activos principalmente a través de la tasa de política y de las expectativas de inflación y de tasa de interés.

Palabras clave: política monetaria, teoría de las expectativas puras, Modelo de Vector de Corrección de Errores.

JEL: C39, E52, D84

\section{Monetary policy and asset prices: An analysis from the interest rate for Colombia from 2003 to 2010}

\section{ABSTRACT}

The main purpose of this paper is to analyze the impact of the Colombian monetary policy on the price of fixed income securities for the 2003-2010 period. It also seeks to verify that the process of transmission is compatible with the arguments of the pure expectations theory.

In order to analyze the relationship between the short and long term TES (the name for the financial securities issued by the Colombian government) and the monetary policy, we apply an econometric model of vector error correction (VEC) using as the dependent variable the return of bonds with maturity terms of either 6 months, and 1, 5 and 25 years; and as explanatory variables we propose the interbank rate, inflation rate and the forward rate. The main results indicate that the Colombian monetary policy affects the price of these assets primarily through its interest rate referential policy, as well as expected inflation and interest rates.

Keywords: monetary policy, pure expectations theory, Vector Error Correction Model. 


\section{Política monetária e preço de ativos: uma análise a partir da taxa de juros para a Colômbia de 2003 a 2010}

\section{RESUMO}

O objetivo principal deste trabalho é analisar a incidência da política monetária colombiana nos preços dos ativos de renda fixa para o período 2003-2010 e comprovar que a via que esta segue se assemelha ao que a teoria tradicional propõe ao tomar como referência a teoria de expectativas puras.

Com o objetivo de analisar as relações de curto e longo prazo entre os Títulos de Tesouraria (TES) e a política monetária, desenvolve-se um modelo econométrico de Vetor de Correção de Erros (VEC) tomando como variável explicada o retorno de bônus com prazo a 6 meses, 1,5 e 25 anos, e como variáveis explicativas a taxa interbancária, a inflação e a taxa forward. Os principais resultados obtidos indicam que a política monetária colombiana incide nos preços desses ativos principalmente por meio da taxa de política e das expectativas de inflação e de taxa de juros.

Palavras-chave: política monetária, teoria das expectativas puras, Modelo de Vetor de Correção de Erros (VEC). 
POLÍTICA MONETARIA Y PRECIO DE ACTIVOS:

\section{INTRODUCCIÓN}

Las decisiones de política monetaria son muy importantes para la economía de cualquier país, ya que afectan todos los mercados incluyendo el de renta fija, el cual ha venido ganando importancia en la economía nacional al ser una alternativa de inversión. Por ello, es fundamental considerar el efecto que tienen las decisiones de política monetaria sobre el precio de estos activos financieros.

Diversos estudios realizados con diferentes metodologías, tanto a nivel nacional como internacional, dan cuenta de que en efecto las decisiones de política perturban el precio de los activos al tener relación directa con su retorno y establecen que la variación del rendimiento depende fundamentalmente de las expectativas sobre la tasa de política y la inflación.

En este sentido, el objetivo principal del presente trabajo es analizar la incidencia de la política monetaria colombiana en los precios de los activos de renta fija y comprobar si la vía que esta sigue se asemeja a lo que plantea la teoría tradicional. Para alcanzar estos objetivos se realiza en primera instancia una revisión literaria referente a cómo la política monetaria afecta la oferta y demanda de bienes financieros; luego se procede con el análisis desde la curva de rendimiento desarrollando la parte matemática de la teoría, se sigue con la especificación del modelo para Colombia y finalmente se desarrolla la evidencia empírica seguida de las conclusiones.

\section{REVISIÓN DE LITERATURA}

El medio directo por el cual la política monetaria perturba a la economía es a través de los mercados financieros, así, cuando las tasas de interés varían, especialmente las de corto plazo, se altera el retorno y precio de los activos financieros. Varios autores han demostrado que los precios de estos activos responden a las intervenciones de política monetaria. Entre los primeros estudios está el de Cook y Hahn (1989). Estos autores tomaron el mercado de Estados Unidos entre el periodo de 1974-1979 y analizaron la reacción del rendimiento de los bonos el día después en que el Banco cambió su tasa de política y encontraron que un cambio en 100 puntos básicos en la tasa de política lleva a un cambio de 55 puntos básicos en la tasa de los bonos del tesoro con tres meses de maduración, lo que causa un cambio de 10 puntos en bonos con vencimiento a 30 años.

Pakko (1996) considera que las acciones de política monetaria afectan la oferta y la demanda de bienes financieros, pues modifican las expectativas del público acerca de los movimientos de política monetaria. Para probar su tesis realizan el mismo estudio de Cook y Hahn en Estados Unidos para el periodo entre 1994-1996 y miden la respuesta del retorno de los bonos federales a cambios específicos en la política monetaria. De modo general, los autores hallan que las acciones de la FED se anticipan en parte a cambios de la política monetaria y este comportamiento es explicado por las expectativas del mercado sobre las acciones futuras de política, como lo revela el mercado de títulos del tesoro y de futuros de fondos federales.

Vickers (1999) introduce en la relación precio y política monetaria el concepto de expectativas estableciendo que los precios de los activos pueden afectar la política monetaria de dos formas conceptualmente distintas: por medio del objetivo que la política monetaria sigue o por medio de la información que los responsables de política observan. Gilchrist y Leahy (2002) también demostraron que, dada la relación positiva entre los precios de los activos con la economía real, un aumento de los precios genera presiones sobre la producción y la inflación y para eliminarlas el Banco Central eleva las tasas de interés y frena así el aumento del precio de los activos.

Con el mismo objetivo, Rigobon y Sack (2004) desarrolla un estimador que identifica la respuesta de los precios de los activos basados en la heterocedasticidad de la política monetaria. Para esto calcula la varianza de los shocks de política y supone que deberá ser mayor en los días de reuniones del Banco Central. Los autores demostraron que el cambio en la varianza de los shocks de política en 
esas fechas es suficiente para medir la capacidad de respuesta de los precios de los activos a la política monetaria, con lo cual se muestra que un aumento a corto plazo de las tasas de interés implica una disminución de los precios de los activos.

Mediante una metodología diferente, Loannidis y Kontonikas (2008) basados en la teoría de flujos de caja consideran que la política monetaria juega un papel importante en la determinación del rendimiento del activo, ya sea influyendo en las expectativas acerca de la actividad económica o mediante la alteración de la tasa de descuento utilizada por los participantes del mercado. Geromichalos, Licari y Suárez-Lledó (2007), apoyados en las propiedades de un modelo monetario donde se valora un activo por su tasa de rendimiento y su nivel de liquidez, muestran que la conexión de política monetaria y precio de los activos se da a través del dinero; así, cuando el Banco Central permite que la tasa de crecimiento del dinero crezca a una tasa más alta aumenta el nivel de inflación y de esta manera se reduce el retorno del dinero.

Aunque algunos de estos trabajos consideran las expectativas como canal importante de la transmisión de política al precio de los activos, no profundizan en la teoría de la curva de rendimientos, la cual permite ver claramente cómo las decisiones de política monetaria influyen en el mercado financiero. Si bien Rigobon (2004) introduce en su análisis la estructura a plazos de la tasa de interés, en sus resultados no resalta el papel de las expectativas en el canal de transmisión, pues da relevancia a los movimientos de la tasa, $y$ encuentra que un aumento en la tasa de interés de corto plazo se traduce en una disminución de precios de los activos y en un desplazamiento hacia arriba de la curva de rendimientos, el cual se hace más pequeño a mayores plazos.

En Colombia el Banco de la República ha realizado varios estudios sobre precio de activos y política monetaria, entre estos esta "Precios de los Activos y Política Monetaria" (Banco de la Republica, 2006), donde se discute sobre cuál es el papel que debe asumir el banco ante burbujas especulativas o desajustes financieros. Como en otras economías, en Colombia el Banco de la República afecta el precio de los activos vía tipo de interés; sin embargo, la autoridad monetaria no puede reaccionar ante cambios en los precios de los activos modificando sus tasas de interés de política (Banco de la Republica, 2006) pues podría desajustar la inflación afectando la economía real e incumpliendo su principal objetivo que es la estabilidad de precios.

En el mismo año, Arango (2006) hace un análisis desde la teoría de la curva de rendimientos desde la hipótesis de expectativas, comprobando teóricamente que los movimientos de la tasa de subastas de expansión del Banco de la República afectan la estructura a plazo de las tasas de interés y por lo tanto las tasas de retorno del mercado secundario de deuda, ya que los agentes pueden prever las medidas que aplicará la autoridad monetaria. Por tal razón, el banco tiene la capacidad de afectar las expectativas inflacionarias y el valor de los activos de la economía en Colombia.

Por otro lado, los resultados econométricos basados en el modelo de Cook y Hahn (1989) no coinciden con la interpretación teórica, puesto que son contrarios a los esperados dado que a corto plazo no se encontró ninguna conexión entre la tasa de intervención y el mercado secundario de la deuda. Una de las posibles explicaciones que dan estos autores acerca de dicha contradicción es que para el caso de Colombia tal vez no se cumple la hipótesis de expectativas ya que el uso de datos de frecuencia diaria no son los adecuados para ilustrar los cambios de política en el mercado de deuda o por el contrario el banco goza de credibilidad.

En 2010, el emisor realizó un estudio de transmisión de la tasa de política a las tasas del mercado y se encontró que en lo corrido de 2009 las tasas de los TES entre 1 y 2 años cayeron 566 puntos básicos, entre 2 y 5 años cayeron 360 pb. y 248 pb. para TES entre 5 y 15 años. Una conclusión por destacar del trabajo es que "la valorización general de los TES, especialmente los de corto plazo, se presentó a medida que los agentes incorporaron las sucesivas disminuciones de las tasas de referencia por parte del Banco de la República. La reducción de las tasas también estuvo relacionada con los 
POLÍTICA MONETARIA Y PRECIO DE ACTIVOS:

datos de inflación que se dieron a conocer en el transcurso del año, y resultaron inferiores a los esperados" (Banco de la República, 2010, p. 340).

\section{ANÁLISIS DESDE LA CURVA DE RENDIMIENTOS}

De Gregorio (2007) afirma que: "La política monetaria incide en la economía primordialmente desde los mercados financieros. El Banco Central, al alterar las tasas de interés de corto plazo, cambia el rendimiento y el precio de los activos financieros". Para interpretar la reacción de los precios de los activos cuando el Banco anuncia su política se hace necesario observar el comportamiento de las principales variables de política monetaria que los afecta. Estas variables son la tasa de interés y las expectativas, las cuales son el mecanismo de transmisión de política monetaria en Colombia. Para Melo (2010) una manera de obtener información sobre la actuación presente y futura de estas variables es mediante el uso de la teoría de la estructura a plazos de los tipos de interés (ETTI).

La ETTI se construye con bonos del Estado y dado que no tienen riesgo de insolvencia son bastante líquidos y sus precios reflejan la información disponible en el mercado, lo cual permite predecir las expectativas de tasa de interés, inflación y deuda pública (Chacón, 2004). En este sentido, la estructura a plazos es definida como la relación existente entre las tasas de interés a corto y a largo plazo, y a la vez se convierte en fuente de información sobre las expectativas de inflación y tasa de interés (Melo, 2010).

Su gráfica, también llamada curva de rendimientos, muestra la reciprocidad entre el rendimiento de bonos de la misma calidad crediticia pero con diferente vencimiento (Fernandez, 2003) y su pendiente o forma es explicada por dos teorías: la teoría de expectativas compuesta por la teoría de hábitat preferido, preferencia de liquidez y expectativas puras, y la teoría de la segmentación del mercado.

La teoría de expectativas puras constituye el pilar del desarrollo de este estudio, pues permite interpretar con facilidad la curva de retornos y observar las expectativas de tasa de interés y de inflación, variables determinantes en la transmisión de la política monetaria al precio de los activos. La hipótesis de expectativas puras considera que la forma de la curva de rendimientos está dada por lo que los agentes esperan sobre la evolución de tasas de interés; en este sentido, "las tasas de largo plazo muestran cuáles son las expectativas de los agentes de mercado sobre los futuros valores de las tasas de corto plazo" (Cumsille, 2009, p. 10).

Matemáticamente, la hipótesis de expectativas se resume en la siguiente expresión:

$E_{t} r^{1}{ }_{t+k}=f_{t}^{k}$

Donde:

$E_{t}$ : Es el operador de expectativas.

$r_{t}:$ Tasa de interés.

$f_{t}:$ Tasa forward.

Dada la expresión [1], Cumsille (2009) afirma que la política monetaria al modificar la tasa de interés de corto plazo puede alterar las tasas de largo plazo, en la medida en que la política afecte las expectativas de las tasas de corto plazo. Para observar el valor esperado de las tasas de corto plazo, la teoría de expectativas supone que la expectativa de la tasa de interés futura es igual a la tasa forward, tasa existente implícitamente entre dos tasas spot de diferentes periodos y la cual es construida a partir de la tasa spot. En este sentido, es posible hablar de la tasa forward a un año dentro de dos años refiriéndose a la tasa spot que existirá en dos años (Cadavid, 2008).

La demostración matemática del supuesto de esta teoría la ofrecen Bodie y Kane (2009) igualando el retorno total de dos estrategias de inversión de $\mathrm{n}$ años: la primera consiste en comprar y mantener un bono cero cupón de $\mathrm{n}$ años a un rendimiento de $\left(1+y_{n}\right)^{n}$; la segunda estrategia es comprar un bono de $\mathrm{n}-1$ años $\mathrm{y}$ al final del periodo reinvertir el mismo dinero en un bono de igual vencimiento, lo que arroja un retorno de $\left(1+y_{n-1}\right)^{n-1} *\left(1+r_{n}\right)$. 
Igualando los retornos se obtiene que:

$$
\left(1+y_{n}\right)^{n}=\left(1+y_{n-1}\right)^{n-1} *\left(1+r_{n}\right)
$$

Dada la curva de retorno observada, se resuelve [2] para $\left(1+r_{n}\right)$.

$$
\left(1+r_{n}\right)=\frac{\left(1+y_{n}\right)^{n}}{\left(1+y_{n-1}\right)^{n-1}}
$$

El numerador es el factor de crecimiento de la inversión en un bono de $\mathrm{n}$ años hasta su maduración y el denominador es el factor de crecimiento de la inversión en el bono de n-1 años. Como la última inversión dura un año más, la razón representa el retorno en un año de un bono de n-1 años de vencimiento.

Bodie y Kane (2009) plantean que, dado que la tasa de interés futura es incierta, es posible especular su valor esperado y por tanto es llamada tasa forward, y $f$ es la tasa de corto plazo futura. Así, [3] toma la forma:

$\left(1+f_{n}\right)=\frac{\left(1+y_{n}\right)^{n}}{\left(1+y_{n-1}\right)^{n-1}}$

[4] puede expresarse como:

$$
\left(1+y_{n}\right)^{n}=\left(1+y_{n-1}\right)^{n-1} *\left(1+f_{n}\right)
$$

La ecuación [4] permite estimar la tasa forward para varios periodos; por tanto, [5] puede reescribirse como:

$$
y_{n}=\frac{1}{n}\left[y_{n}+f_{n}^{1}+f_{n}^{2}+f_{n}^{3}+\cdots+f_{n}^{n-1}\right]^{1 / n}
$$

Esta ecuación dice que la tasa de largo plazo (spot) es igual al promedio entre la tasa actual y todas las tasas forward.

Tras aplicar en [6] la hipótesis de la teoría de expectativas puras, la ecuación fundamental de este estudio será:

$$
\begin{aligned}
& y_{t}^{n}=\frac{1}{n}\left[y_{t}^{1}+E_{t} r_{t+1}^{1}+E_{t} r_{t+2}^{1}\right. \\
& \left.+E_{t} r_{t+3}^{1}+\cdots+E_{t} r_{t+n-1}^{1}\right]
\end{aligned}
$$

La expresión [7] también dice que la tasa de interés de largo plazo es el promedio de las tasas cortas desde hoy a término. En este sentido, si la expectativa de la tasa de interés de corto plazo $\left(E_{t} r_{t+n-1}\right)$ es mayor que la tasa de rendimiento de hoy la pendiente de la curva es positiva y se espera que las tasas de interés de corto plazo suban en el futuro respecto al nivel de hoy y que la tasa de largo plazo siga subiendo.

Generalmente la ETTI tiene pendiente creciente, pero también existen curvas con pendiente negativa donde las tasas de largo plazo son menores a la tasa de corto plazo, lo que muestra expectativas a la baja de la tasa de interés e inflación. La curva también puede ser horizontal; en este caso, las tasas no cambian como función del plazo de vencimiento (Sachs y Larrain, 1994), es decir, las tasas de interés se mantendrán en su nivel en el tiempo. Finalmente, una curva jorobada (creciente y luego decreciente) se da cuando los retornos del corto y largo plazo son los mismos, pero el rendimiento de mediano plazo varía (Castrillón y Ramírez, 2010).

Fabozzi (2010) ilustra la teoría de las expectativas puras considerando cómo las expectativas de un incremento de las tasas de corto plazo afectan el comportamiento de varios participantes en el mercado. El autor asume que inicialmente la ETTI es plana y supone que un anuncio de una política restrictiva conduce a los agentes a esperar que las tasas de interés se incrementen.

Desde estas condiciones se dan tres escenarios simultáneamente: en el primer escenario los agentes interesados en inversiones de largo plazo no desean comprar bonos de largo plazo pues esperan que la curva de rendimientos aumente y lleve así a una pérdida del capital debido a que el precio de los bonos cae; en lugar de esto, los agentes invertirán su dinero en obligaciones de corto plazo y esperarán a que el rendimiento aumente 
para reinvertir sus fondos. El segundo escenario consiste en que los especuladores anticipan la caída del precio de los bonos de largo plazo y por lo tanto los venderán $y$, al igual que en el primer escenario los agentes reinvertirán en obligaciones de corto plazo. En el tercer escenario los prestatarios estarán motivados a endeudarse, dado que en el tiempo los préstamos serán más caros y por consiguiente prefieren adquirir bonos de largo plazo (Fabozzi, 2010).

Por lo tanto, estas decisiones llevan a que aumente la oferta o disminuya la demanda de bonos de largo plazo incrementando la demanda de obligaciones de corto plazo. Con el fin de incentivar la demanda de títulos de largo plazo, Fabozzi (2010) explica que debe darse un incremento en el rendimiento de largo plazo con respecto al corto plazo, por analogía, decisiones de política que lleven expectativas a la baja de la tasa de interés resultarán en una curva de rendimientos de pendiente negativa.

La teoría de la hipótesis de expectativas puras muestra de una manera clara y precisa cómo las decisiones de política monetaria modifican el precio de los activos al incidir directamente en la tasa de interés y en las decisiones de los agentes. En consecuencia, es posible construir un modelo econométrico para el caso colombiano que se centre en el estudio del efecto de la política monetaria sobre el precio de los activos y logre abarcar las interrelaciones entre las variables y sus relaciones de largo y corto plazo.

\section{ESPECIFICACIÓN DEL MODELO PARA COLOMBIA}

El análisis teórico aquí desarrollado plantea que la política monetaria afecta principalmente el retorno de los TES a través de las expectativas de tasa de interés y de inflación. Con el objetivo de verificar esta hipótesis para Colombia en periodos mensuales comprendidos entre $2003-2010$ se especifica una función donde el rendimiento de los TES está explicado por la tasa de interés de política monetaria que por simplicidad técnica será la tasa interban- caria (TIB), variable proxy de la tasa de intervención del Banco de la República, tasa de inflación y las expectativas de tasa de interés y de inflación.

Esta especificación se deriva de la teoría de expectativas puras expuesta en la cual las expectativas de tasa de interés y de inflación están dadas por la tasa forward. Así, la especificación toma la forma:

$r T E S=\beta_{1}+\beta_{2} f+\beta_{3} T I B+\beta_{4} I N F$

Donde:

rTES: es el rendimiento de los bonos con vencimiento a seis meses y vencimiento a 1, 5 y 25 años.

$f$ : es la tasa forward de los bonos con plazos diferentes y se toma como medida de expectativa de mercado para la tasa de política y de inflación.

TIB: es la tasa interbancaria y recoge de forma rápida y continua los cambios de corto a largo plazo de la tasa de referencia.

INF: es la tasa de inflación e indica el crecimiento generalizado de los precios de bienes, servicios y factores productivos en la economía.

En general, lo que se espera obtener con el uso del VEC es que la aplicación de una política monetaria contractiva afecte de manera positiva el rendimiento de los TES con diferente maduración y que, dada la relación entre retorno y precio, incida de manera negativa sobre los precios de estos bonos. Desde la hipótesis de expectativas puras, el aumento de la tasa de política ha de venir acompañado en corto plazo por una variación positiva en las expectativas de los agentes sobre la inflación y la misma tasa de interés; sin embargo, en el largo plazo se espera que se retorne de nuevo a la relación de equilibrio que mantiene las variables.

Con el fin de representar adecuadamente la dinámica entre estas variables, se utiliza como herramienta econométrica un VEC por dos razones 
teóricas esenciales que justifican el uso de este método. Primero, el rendimiento de los TES y la tasa de intervención al ser tasa de interés están estrechamente relacionados por el mecanismo de transmisión de la política monetaria, y segundo porque en Colombia la política monetaria es manejada por una regla con objetivo de inflación; por lo tanto, la tasa de política y su expectativa resulta de la creencia del comportamiento futuro de la inflación evidenciando una fuerte correlación entre la tasa forward (variable que mide las expectativas), la tasa interbancaria y la inflación. Por ello, se debe considerar la existencia de cointegración entre las variables y pensar en la posible presencia de equilibrio de largo plazo. EI VEC permite considerar la dinámica entre corto y largo plazo existente entre las variables, y se convierte en una buena herramienta para lograr el objetivo de este trabajo.

Para estimar el VEC se aplica el enfoque de cointegración de Johansen, el análisis parte de la especificación de un Vector Autorregresivo (VAR), definido por:

$$
\begin{aligned}
& X_{t}=m+\prod_{1} X_{t-1}+\prod_{2} X_{t-2} \\
& +\cdots+\prod_{t-k} X_{t-k}+\epsilon_{t}
\end{aligned}
$$

Donde:

$X$ : es la matriz de información que contiene a rTES, $f$, TIB y INF.

П: es la matriz de coeficientes de rezagos.

La representación adecuada del enfoque de cointegración de Johansen es un VEC, donde [9] se puede representar como:

$$
\Delta X_{t}=\Gamma(\mathrm{L}) \Delta X_{t}+\prod X_{t-1}+\epsilon_{t}
$$

Donde $\Gamma(\mathrm{L})$ es el operador de rezagos dado por:
$\Gamma(\mathrm{L})=\prod_{i+1}+\prod_{i+1}+\cdots+\prod_{k}$

$\Pi=\prod_{1}+\prod_{2}+\cdots+\prod_{k}$

Como primer paso se determinó el rezago óptimo de cada VAR usando el estadístico LR (Relación de Probabilidad) y los criterios de información AIC (Akaike), SC (Schwarz), HQ (Hannan Quinn) y FPE (Predicción Final del Error). También se usó como prueba auxiliar la gráfica del polinomio autorregresivo y la prueba de raíces unitarias.

Para la mayoría de TES las pruebas sugieren un rezago de $\mathrm{k}=4$; sin embargo, para TES con maduración de 10 años el rezago optimo es $k=1$; de esta manera, se estima un VAR (4) para el rendimiento de TES de 6 meses, 1, 5 y 25 años. Inicialmente se incluyó el rendimiento de los TES con vencimiento a 10 años; no obstante, no se tiene en cuenta en el análisis dado que el VAR estimado para este presenta relaciones espurias y por tanto la inferencia no es correcta.

Para probar la pertinencia de las estimaciones y no tener relaciones espurias, se verifica que los residuos generados por cada VAR sigan un proceso estacionario. De igual manera, se verifica que no presenten problemas de normalidad, autocorrelación y heterocedasticidad.

Una vez obtenidas las pruebas de los residuos se contrasta si existen relaciones de cointegración en cada VAR estimado. La posible existencia de una relación de largo plazo indica los movimientos de los rendimientos de los TES con diferentes vencimientos a causa de la política monetaria aplicada. Para contrastar la existencia o no de relaciones de cointegración se usa el procedimiento de Johansen. Los resultados de este test se presentan en la tabla 1.

La tabla 1 muestra que para el rendimiento del TES con diferente vencimiento puede encontrarse un vector de cointegración, es decir, es posible identificar una relación estable de largo plazo en las variables consideradas. La existencia de equilibrio implica que las variables se mueven de forma compacta en el tiempo (Galindo, 1997). 
Tabla 1.

Prueba de cointegración de Johansen

\begin{tabular}{|c|c|c|c|c|c|}
\hline \multicolumn{6}{|c|}{ VAR TES 6 meses } \\
\hline Data Trend & None & None & Linear & Linear & Quadratic \\
\hline \multirow[t]{2}{*}{ Test Type } & No Intercept & Intercept & Intercept & Intercept & Intercept \\
\hline & No Trend & No Trend & No Trend & Trend & Trend \\
\hline Trace & 1 & 2 & 2 & 1 & 1 \\
\hline Max - Eig & 1 & 0 & 1 & 0 & 0 \\
\hline \multicolumn{6}{|c|}{ Unrestricted Cointegration Rank Test (Trace) } \\
\hline Hypothesized & & Trace & 0.05 & & \\
\hline No. Of CE (s) & Eigenvalue & Statistic & Critical Value & Prob. ** & \\
\hline None * & 0.260059 & 48.70911 & 40.17493 & 0.0056 & \\
\hline At most 1 & 0.136098 & 21.30131 & 24.27596 & 0.1133 & \\
\hline At most 2 & 0.075950 & 7.988378 & 12.32090 & 0.2376 & \\
\hline At most 3 & 0.008756 & 0.800342 & 4.129906 & 0.4274 & \\
\hline \multicolumn{6}{|c|}{ VAR TES 1 año } \\
\hline Trace & 1 & 2 & 2 & 1 & 1 \\
\hline Max - Eig & 1 & 2 & 2 & 0 & 0 \\
\hline None * & 0.264544 & 47.15114 & 40.17493 & 0.0086 & \\
\hline At most 1 & 0.122813 & 19.19003 & 24.27596 & 0.1917 & \\
\hline At most 2 & 0.072323 & 7.265796 & 12.32090 & 0.2994 & \\
\hline At most 3 & 0.00476 & 0.434232 & 4.129906 & 0.5734 & \\
\hline \multicolumn{6}{|c|}{ VAR TES 5 año } \\
\hline Trace & 1 & 2 & 3 & 3 & 4 \\
\hline Max - Eig & 1 & 1 & 1 & 1 & 1 \\
\hline None * & 0.303445 & 52.30433 & 40.17493 & 0.0020 & \\
\hline At most 1 & 0.157154 & 19.39795 & 24.27596 & 0.1824 & \\
\hline At most 2 & 0.040011 & 3.839621 & 12.32090 & 0.7339 & \\
\hline At most 3 & 0.001359 & 0.123766 & 4.129906 & 0.7719 & \\
\hline \multicolumn{6}{|c|}{ VAR TES 25 año } \\
\hline Trace & 0 & 1 & 1 & 1 & 2 \\
\hline Max - Eig & 0 & 1 & 1 & 0 & 0 \\
\hline None * & 0.278189 & 57.51017 & 54.07904 & 0.0239 & \\
\hline At most 1 & 0.147009 & 27.84485 & 35.19275 & 0.2483 & \\
\hline At most 2 & 0.118053 & 13.37532 & 20.26184 & 0.3346 & \\
\hline At most 3 & 0.021132 & 1.943629 & 9.164546 & 0.7889 & \\
\hline
\end{tabular}

Fuente: Elaboración de los autores. Datos obtenidos del Banco de la República de Colombia. 
Estas relaciones de equilibrio son llamados mecanismos de corrección del error y expresan los mecanismos y magnitudes del ajuste de los agentes, en la medida en que fuerzan a las variables a regresar al equilibrio ante la presencia de desajustes (Galindo, 1997). En este caso, los coeficientes de los vectores de cointegración reflejan las reglas que siguen los agentes económicos para mantener el rendimiento de bonos en su nivel de equilibrio en cuanto el emisor modifique directamente la tasa de interés y afecte la inflación y las expectativas de interés y precios.

Tras determinar las relaciones de cointegración en cada VAR se estima el VEC, el cual arroja la ecuación de cointegración para cada VAR estimado, como se puede observar en la tabla 2.

\section{Evidencia empírica}

Para analizar el impacto de la política monetaria sobre el precio de los bonos se realizó un análisis basado en la teoría de expectativas puras que permitió definir tres variables que hacen parte de la política monetaria: tasa interbancaria, tasa de inflación y expectativas de inflación y tasa de interés.
El modelo econométrico empleado se basó en el VEC y sus principales resultados se presentan en la tabla 2; en general, los resultados confirman las relaciones teóricas esperadas entre las variables.

Se puede observar que existe relación positiva entre el rendimiento de los TES con diferente maduración y su tasa forward respectiva. Además, se observa que a medida que aumenta el vencimiento del bono la tasa forward tiene un mayor efecto marginal sobre su rendimiento. Por esta razón, se podría considerar que las expectativas de inflación y tasa de interés juegan un papel importante en la explicación de movimientos en el largo plazo en el retorno del TES y por consiguiente en su precio. Esto quiere decir que los cambios en las expectativas de los agentes afectarán en mayor medida la tasa de rendimiento de bonos de mayor plazo y en menor medida la tasa de los bonos de corto plazo.

Este resultado se explica como consecuencia del riesgo inflación que refleja las expectativas; así, un aumento de las expectativas estará advirtiendo un posible incremento de la inflación. Por tanto, los inversionistas exigirán un mayor rendimiento para compensar la pérdida de poder adquisitivo y el emisor asumirá una política monetaria restrictiva para anclar las expectativas. Sin embargo, se debe

Tabla 2.

Relaciones de cointegración

\begin{tabular}{|c|c|c|c|c|c|}
\hline & TES it (-1) & f(TES it) (-1) & INF (-1) & TIB (-1) & C \\
\hline VAR TES 6 meses & 1.000000 & -0.997475 & -0.228567 & -0.125231 & - \\
\hline Error estándar & & $(0.00123)$ & $(0.02984)$ & $(0.02309)$ & \\
\hline Estadístico t & & {$[-810.300]$} & {$[-7.66012]$} & {$[-5.42368]$} & - \\
\hline VAR TES 1 años & 1.000000 & -1.003811 & -0.936576 & -0.101346 & $(0.07176)$ \\
\hline Error estándar & & $(0.00411)$ & $(0.09114)$ & {$[-1.41237]$} & \\
\hline estadístico t & & {$[-244.460]$} & {$[-10.2765]$} & 1.57833 & \\
\hline VAR TES 5 años & 1.000000 & -1.057361 & -6.624469 & $(0.73003)$ & - \\
\hline Error estándar & & $(0.07170)$ & $(0.86838)$ & {$[2.16200]$} & \\
\hline estadístico t & & {$[-14.7471]$} & {$[-7.62854]$} & -0.263877 & -0.236206 \\
\hline VAR TES 25 años & 1.000000 & -1.0843 & 1.935365 & $(0.21385)$ & $(0.01235)$ \\
\hline Error estándar & & $(0.14131)$ & $(0.31618)$ & {$[-1.23395]$} & {$[-19.1311]$} \\
\hline estadístico t & & {$[-7.67316]$} & {$[6.12045]$} & & \\
\hline
\end{tabular}

Fuente: Elaboración de los autores. Datos tomados del Banco de la República de Colombia. 
POLÍTICA MONETARIA Y PRECIO DE ACTIVOS:

considerar que a medida que se incrementan los años de maduración del bono las expectativas son menos significativas. Esto se debe a que en horizontes de tiempo largo para el agente es difícil formarse expectativas.

Por otro lado, se encuentra que la inflación también tiene la relación esperada con los rendimientos de los TES y sus precios, ya que el efecto positivo de la inflación sobre el rendimiento, al igual que las expectativas, aumenta conforme se incrementa el vencimiento del bono. Esta relación positiva refleja el hecho de que los agentes exigen una mayor tasa de interés nominal dada la pérdida de rendimiento real por causa del incremento inflacionario. Otra explicación a este vínculo está en que el Banco Central, el cual, por cuestión de credibilidad, compensa el aumento de la inflación con incrementos en la tasa de política y por el mecanismo de transmisión entre tasas. Esto resultara en un aumento de los rendimientos de los bonos y en una caída del precio (Jareño, 2007).

La tasa de política monetaria también cumple la relación teórica que se esperaba y dicha relación está explicada por el mecanismo de transmisión existente entre tasas, el cual tiene como canales principales el crediticio y el de las expectativas. La tasa de política afecta las demás tasas de mercado cuando los bancos para satisfacer sus necesidades de liquidez acuden por cupo al Banco Central y transan a la tasa de intervención fijada por el emisor. De esta manera, cuando el banco modifica su tasa genera costos en los fondos de los demás bancos y afecta así la tasa de interés de mercado en el largo plazo y el precio de los bonos (Banco de la República, 2005).

Dos resultados atípicos deben ser mencionados, el primero es la relación negativa entre el rendimiento de los TES a 5 años con la TIB, el segundo es la relación negativa entre el rendimiento de TES a 25 años con la inflación. Esta última relación es inusual, pues los TES son activos indexados o que tienen en cuenta la inflación y una prima por riesgo para determinar su rendimiento.

Al momento de estimar la tasa forward para obtener la medida de expectativas de inflación y tasa de interés se observa que al graficarla esta presenta pendiente positiva y por tanto, se espera que las tasas de corto plazo aumenten en el futuro. Este resultado coincide con la estimación de la tasa forward para Colombia en diferentes investigaciones, las cuales concluyen que la forma de la curva de rendimientos tiene pendiente positiva, considerando que existen expectativas al alza de la inflación y tasa de interés. Una curva de rendimientos de pendiente positiva es considerada como un comportamiento normal, pues generalmente las tasa de corto plazo son menores a las tasas de largo plazo, principalmente por el riesgo de la inflación y la prima por maduración de los activos.

La tabla 2 presenta también los resultados de las estimaciones del VEC para cada TES mostrando que el coeficiente de determinación de cada modelo es pequeño e indicando que la política monetaria explica en menor grado las variaciones de los rendimientos de los TES y, por ende, los cambios en sus precios. El signo positivo de este coeficiente indica que las variables en su conjunto tienen relación positiva con el rendimiento de los precios. Es importante este resultado pues confirma que la política monetaria afecta en sentido directo al retorno de los bonos y por lo tanto, de manera indirecta, sus precios.

\section{CONCLUSIONES}

Este trabajo usa como marco la teoría de expectativas puras y aplica un VEC para establecer la dinámica de corto y largo plazo entre las variaciones de los precios de activos de renta fija y cambios en la tasa de interés de política monetaria. Los principales resultados obtenidos indican que la política monetaria colombiana incide en los precios de estos activos, principalmente a través de la tasa de política, de las expectativas de inflación y de tasa de interés. Dado que los coeficientes de la ecuación de cointegración para el retorno de diferentes TES tienen el signo teórico esperado, es posible señalar que la evolución del precio de los bonos para Colombia tiene un comportamiento similar al expuesto en la teoría. Aunque se hace inusual que no se cumpla 
la relación entre la tasa de política y el rendimiento de TES de cinco años de maduración.

Se evidenció que las expectativas son determinantes en la explicación del comportamiento de los precios de los activos de renta fija de menor maduración. Para plazos mayores se le dificulta al inversionista formarse expectativas acerca de la inflación dada la incertidumbre que implica el mayor plazo. Asimismo, los resultados de la estimación de la tasa forward afirman la credibilidad que el Banco de la República ha construido desde la implementación del esquema de inflación objetivo.
Es claro que este estudio se encuentra limitado a las variables acá incluidas, y por tanto puede ser un análisis incompleto. No solo no se tiene en cuenta el riesgo de interés, sino que también se obvian los demás riesgos asociados, como el riesgo de inflación, de cambio, de reinversión, etc. A pesar de estas limitaciones, el análisis al concentrarse en las variables más importantes de la política monetaria logra demostrar que en efecto las decisiones del emisor modifican el precio de los bonos, principalmente vía tasas de interés y expectativas.

\section{REFERENCIAS}

1. Arango Thomas, L. E., González A., León Díaz, J. J. y Melo Velandia, L. F. (2006). Efectos de los cambios en la tasa de intervención del Banco de la República sobre la estructura a plazo. Borradores de Economía, 424. 31 p. Recuperado de: http://www.banrep.gov.co/docum/ftp/ borra424.pdf

2. Banco de la Republica (2006). Precios de los activos y politica monetaria. Reportes del Emisor: Investigacion e Informacion Económica. 83, 1-6. Recuperado de http://www.banrep.gov.co/sites/ default/files/publicaciones/archivos/83.pdf

3. Banco de la República. (2005). Algunos comentarios sobre la transmisión de la politíca monetaria y el canal del crédito. Bogota: Reportes del Emisor: Investigación e Información Económica. 77, 1-8. Recuperado de http://www.banrep.gov.co/sites/default/files/publicaciones/archivos/77.pdf

4. Banco de la República. (2010). Tasas de interes del Banco de la república y de mercado. Banco de la República: Reportes del emisor: Investigación e información Económica. 128, 1-6. Recuperado de http://quimbaya.banrep.gov.co/documentos/publicaciones/report_emisor/2010/128.pdf

5. Bodie, Z. y Kane, A. (2009). Investiments (8va. ed.). México: McGraw-Hill.

6. Cadavid, J. N. (2008). Cálculo de las tasas de rendimiento de los títulos de tesorería (TES B) en Colombia mediante splines cúbicos. Pereira: Universidad Tecnológica de Pereira.

7. Castrillón, M. R. y Ramírez, A. (2010). La Curva de Rendimientos como un indicador adelantado de la actividad económica, el caso colombiano: Periodo 2001-2009. Medellin: Ecos de economía.

8. Chacón, R. (2004). Estructura temporal de las tasas de interés: curva cupón cero. I Simposio Docentes de Finanzas: Politécnico Grancolombiano, Colombia.

9. Cook, T. y Hahn, T. (1989). The effect of changes in the federal funds rate target on market interest rates in the 1970s. Journal of Monetary Economics, 24(3), 331-351. doi: http://dx.doi. org/10.1016/0304-3932(89)90025-1

10. Cumsille, J. N. (2009). La curva de rendimientos y la política monetaria en la economía chilena. Santiago de chile: Pontificia Universidad Católica de Chile.

11. De Gregorio, J. (2007). Macroeconomía: Teoría y Politicas. México: Pearson.

12. Fabozzi, F. J. (2010). Bond Markets, Analysis, and Strategies (7ma. ed.). México: Pearson. 
13. Fernández, A. D. (2003). Politica Monetaria: Fundamentos y Estrategias. España: Ediciones Paraninfo S.A.

14. Galindo, L. M. (1997). Un modelo econometrico de vectores autorregresivos y de cointegración para la economía mexicana, 1980-1996. Mexico: Economía Mexicana. Nueva Época.

15. Geromichalos, A., Licari, J. M. y Suárez-Lledó, J. (2007). Monetary policy and asset prices. Review of Economic Dynamics, 10(4), 761-779. doi: http://dx.doi.org/10.1016/j.red.2007.03.002

16. Gilchrist, S. y Leahy, J. V. (2002). Monetary policy and asset prices. Journal of Monetary Economics, 49(1), 75-97. doi: http://dx.doi.org/10.1016/S0304-3932(01)00093-9

17. Ioannidis, C. y Kontonikas, A. (2008). The impact of monetary policy on stock prices. Journal of Policy Modeling, 30(1), 30-5.

18. Jareño, F. (2007). Sensibilidad de las acciones españolas ante cambios en los tipos de interés: un estudio desde distintos enfoque. Análisis Financiero, 103, 30-35.

19. Melo, L. y Castro, G. (2010). Relación entre variables macro y la curva de rendimientos. Borradores de Economía, 105, 29 p.

20. Pakko, M. (1996). Monetary policy and financial market expectations: What did they know and when did they know it? In D. Wheelock (ed.) (pp. 19-32), The Federal Reserve Bank of St. Louis Review.

21. Rigobon, R. y Sack, B. (2004). The impact of monetary policy on asset prices. Journal of Monetary Economics, 51, 1553-1575

22. Sachs, J. D. y Larrain, F. (1994). Macroeconomía en la economía global. México: Prentice Hall.

23. Vickers, J. (1999). Monetary policy and asset prices. The Manchester School. 68, 1-22. DOI: 10.1111/1467-9957.68.s1.1 
